\title{
Comparative Study on Effect of General Fatigue on Visual and Auditory Reaction Times
}

\section{Sara Sarhadi*, Majid Kashef}

Department of Exercise Physiology, Faculty of Physical Education and Sport Sciences, University of Shahid Rajaee Teacher Training, Tehran, Iran

\section{A BSTRACT}

Introduction: Several vital body systems, such as visual and auditory systems, can be influenced by fatigue. Each system can be affected based on the complexity of its tasks and the level of involvement in information processing in the brain. The aim of this study was to evaluate the effect of fatigue on visual and auditory reaction times after an exhaustive exercise. Materials and Methods: 15 female students of Shahid Rajaee University participated in this study. Bruce test was used to make general fatigue. The dependent variables include visual and auditory reaction times that compared in fatigue and non-fatigue conditions. Kolmogorov- Smirnov test was used for considering data distribution and Paired $\mathrm{t}$ test was used for considering the differences between groups. Results: The results revealed a significant increase in visual and auditory reaction times in fatigue conditions. However, the data showed that fatigue has the same effect on the visual and auditory reaction times. Conclusion: The results showed that general fatigue influenced equally the visual and auditory systems.

Key words:

1. Fatigue

2. Reaction Time

3. Auditory Fatigue

*Corresponding Author: Sara Sarhadi

E-mail: sara.sarhaddi@gmail.com 
بررسى مقايسةُ اثر خستخى عمومى بر زمان واكنش بينايیى و شنوايى

$$
\text { سارا سرحدى"، مجيد كاشف }
$$

كروه فيزيولوزى ورزشى، دانشكده تربيت بدنى و علوم ورزشى، دانشكاه تربيت دبير شهيد رجايى، تهران، ايران

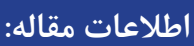

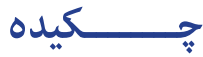

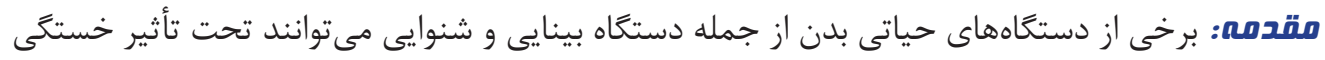

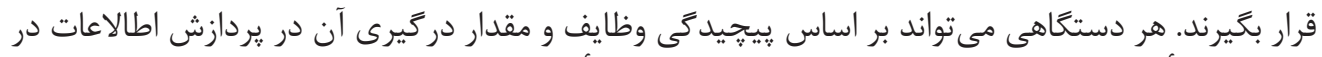

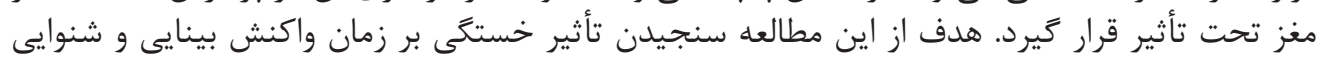

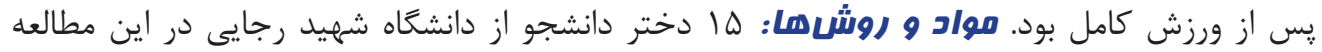

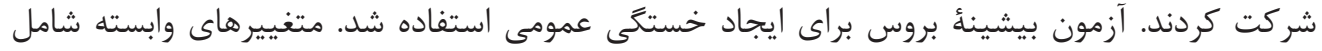

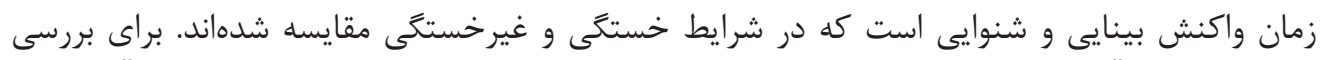

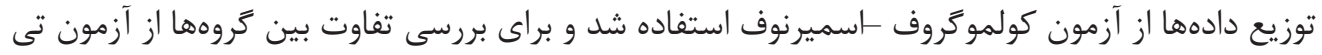

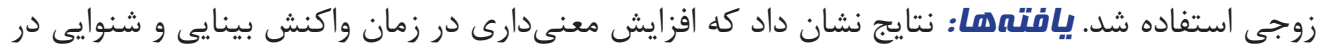

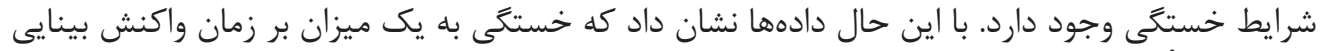

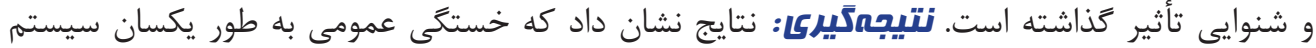
بينايى و شنوايى را تحت تأثير قرار داده است.

\footnotetext{
كليد وازهها: r. r. زمان واكنش

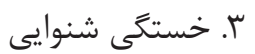




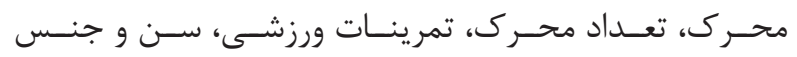

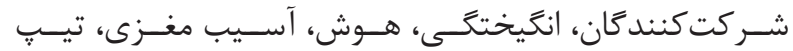

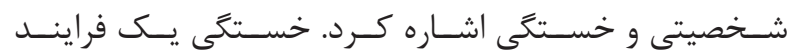

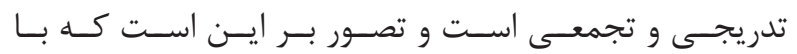

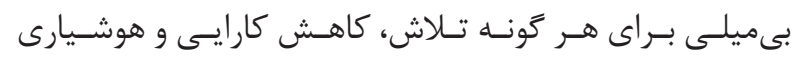

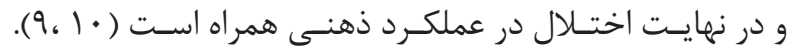

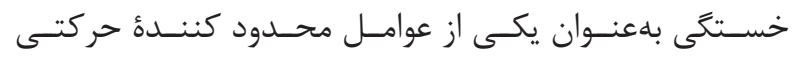

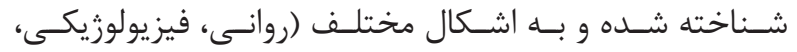

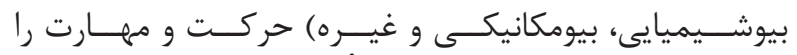

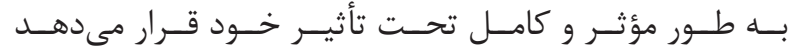

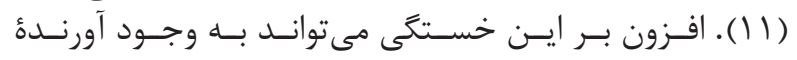

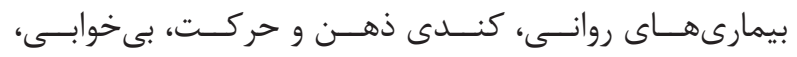

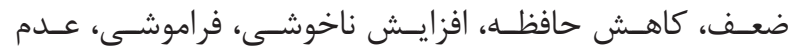

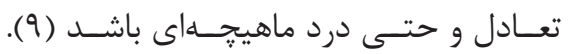

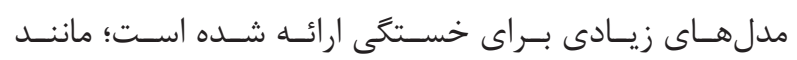

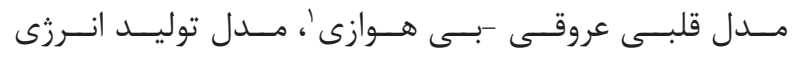

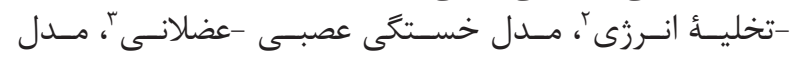

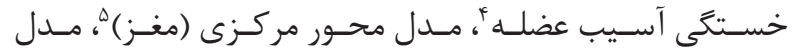

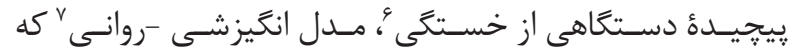

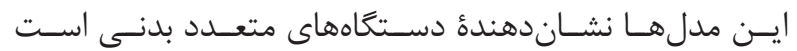

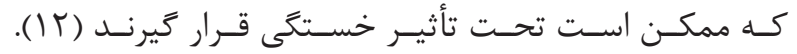

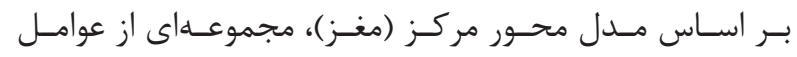

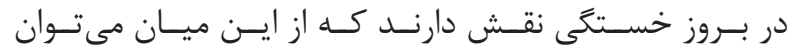

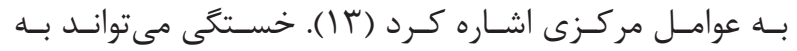

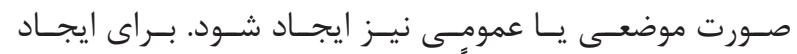

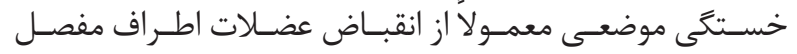

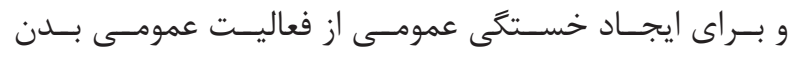

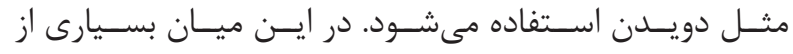

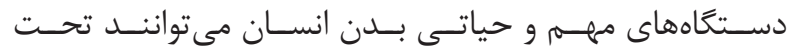

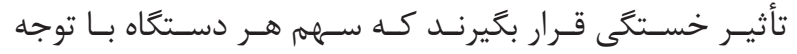

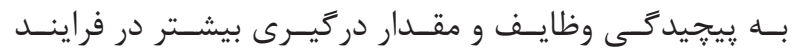

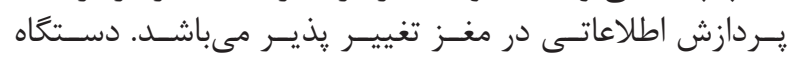

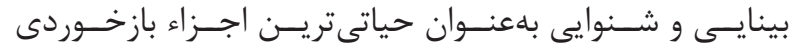

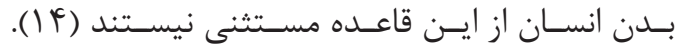

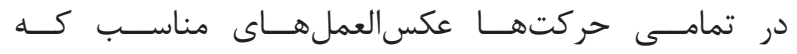

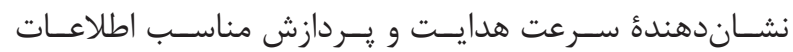

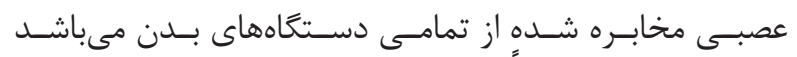

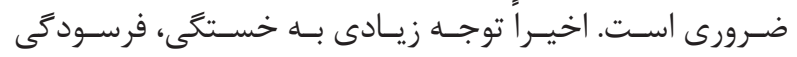

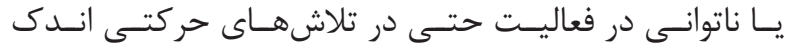

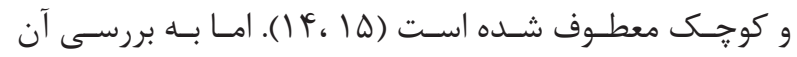

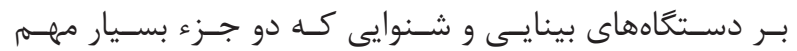

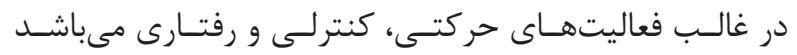

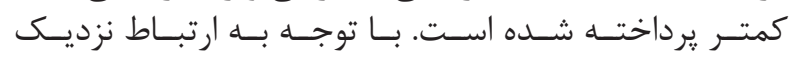

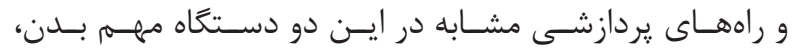

\footnotetext{
${ }^{1}$ Model cardiovascular/anaerobic

${ }^{2}$ Energy supply/energy depletion model

${ }^{3}$ Neuromuscular model of fatigue

${ }^{4}$ Muscle trauma model of fatigue
}

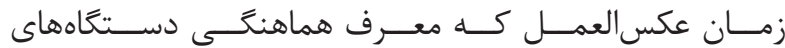

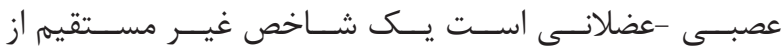

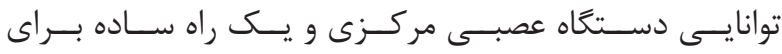

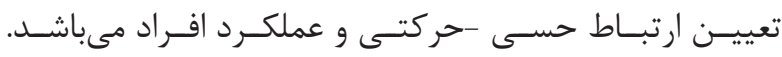

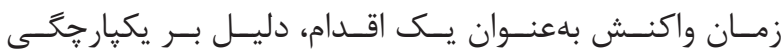

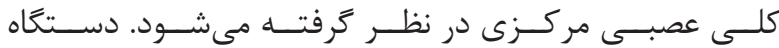

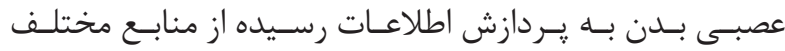

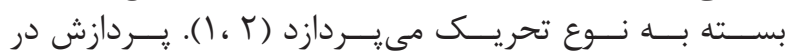

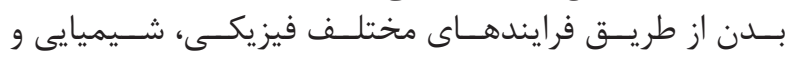

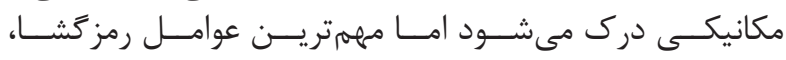

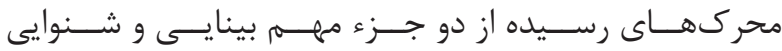

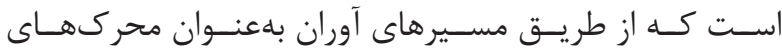

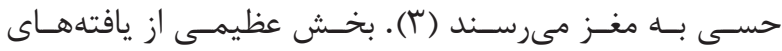

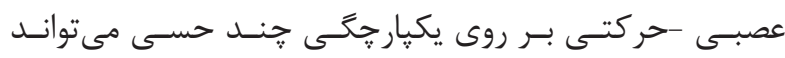

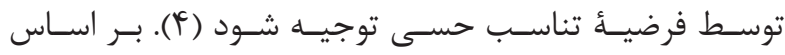

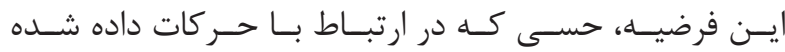

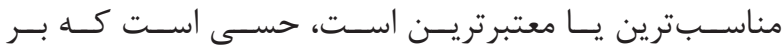

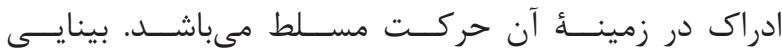

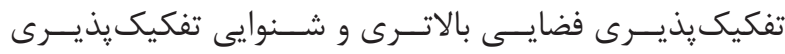

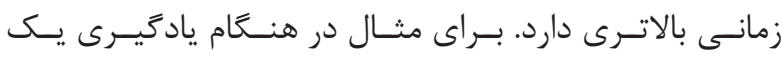

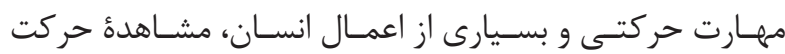

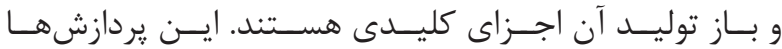

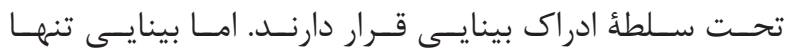

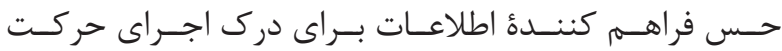

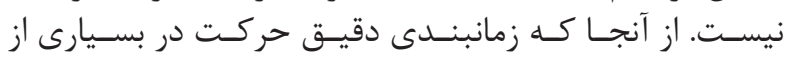

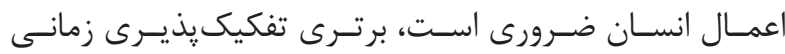

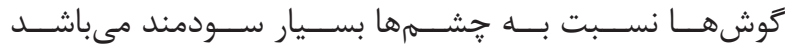

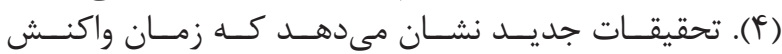

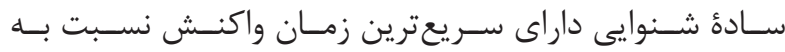

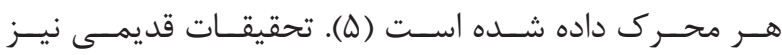

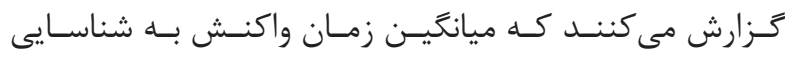

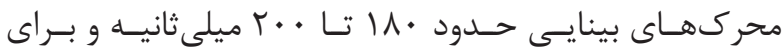

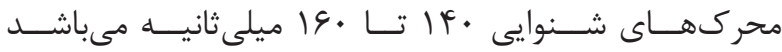

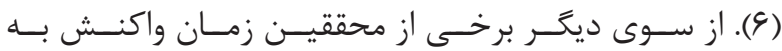

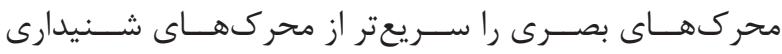

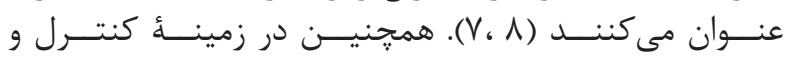

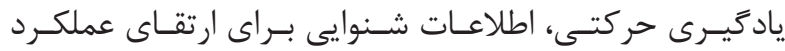

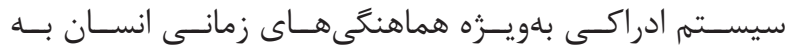

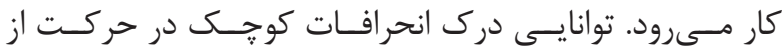

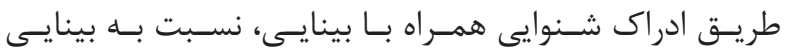

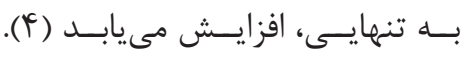

در ايـن ميــان عوامـل مختلفـى بــر زمـان واكنـش تأثيركـــار

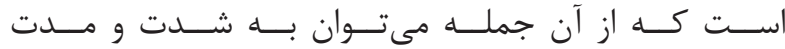

${ }^{5}$ Central governor mode

${ }^{6}$ Complex systems model of fatigue

${ }^{7}$ Psychological/motivational model 


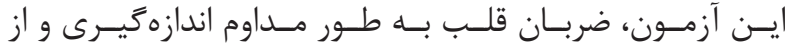

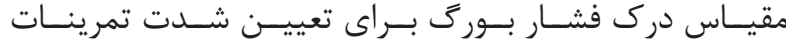

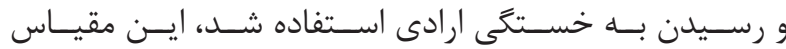

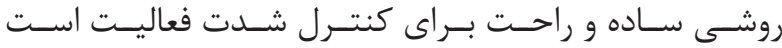

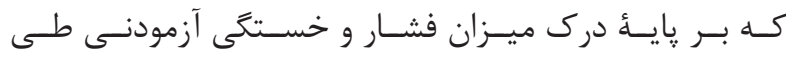

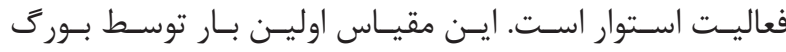

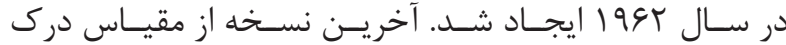

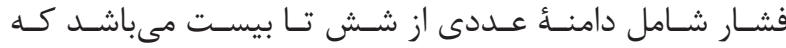

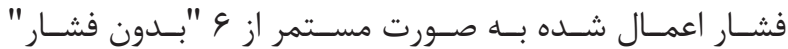

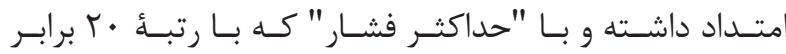

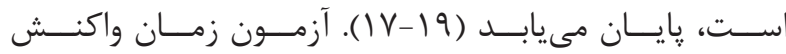

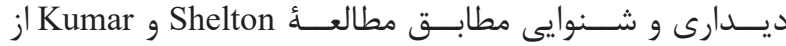

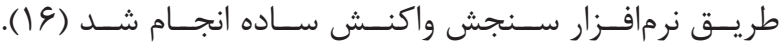

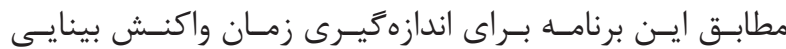

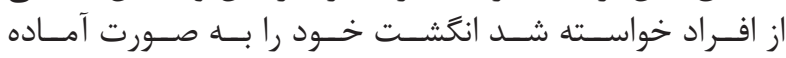

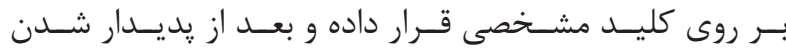

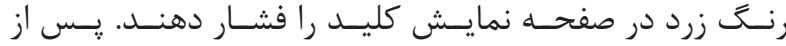

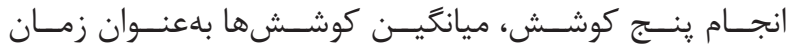

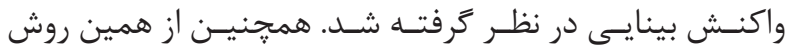

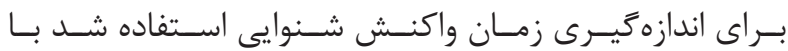

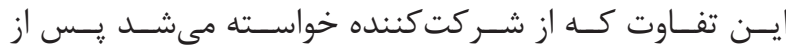

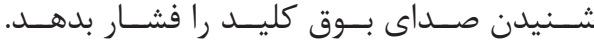

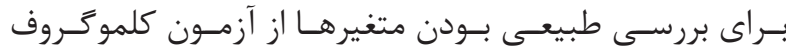

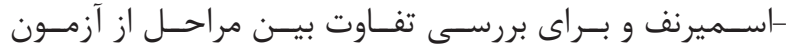

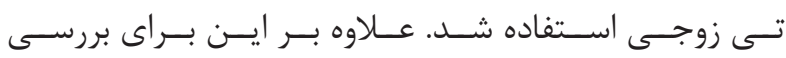

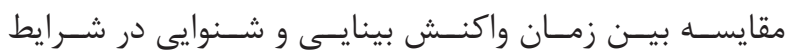

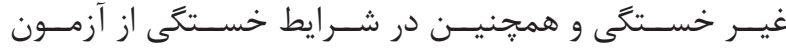

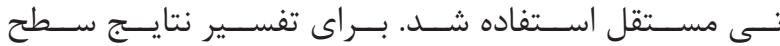

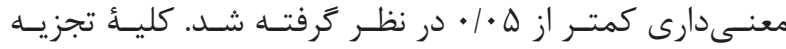

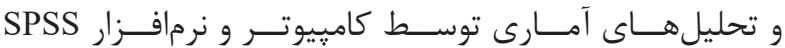

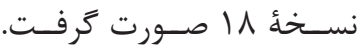

كافتهها

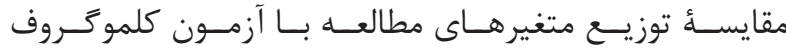

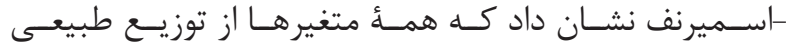

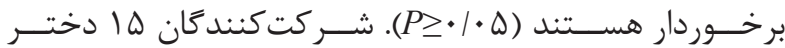

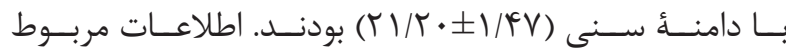

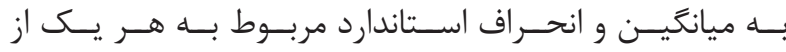

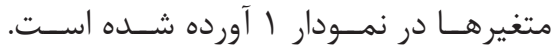

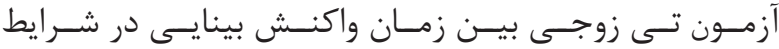

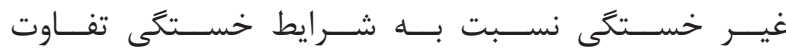

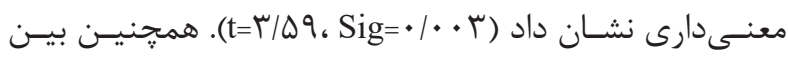

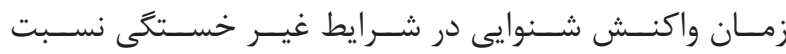

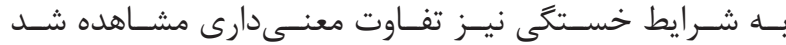

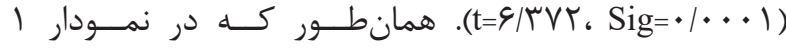

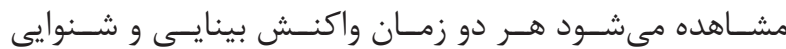

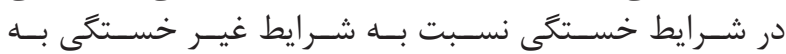

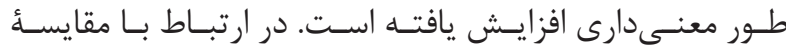

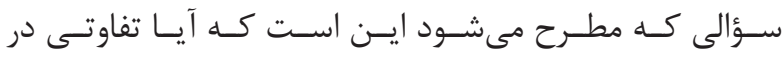

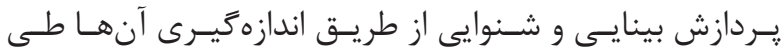

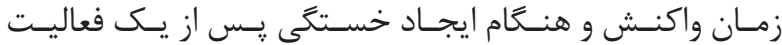

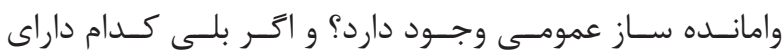

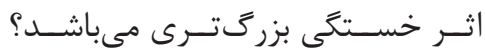

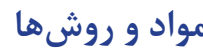

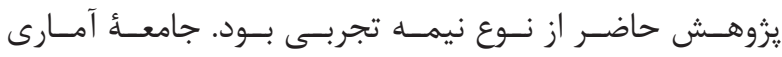

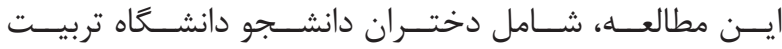

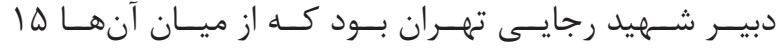

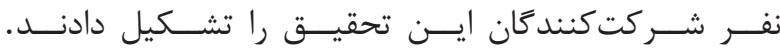

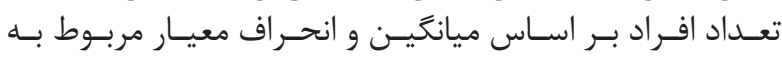

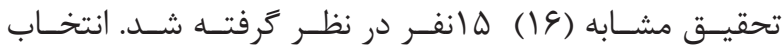

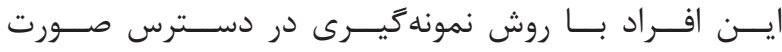

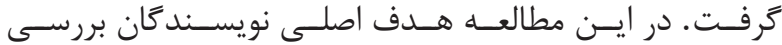

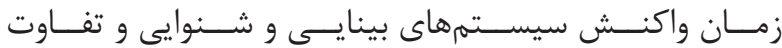

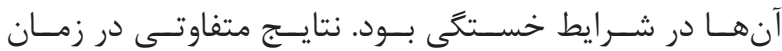

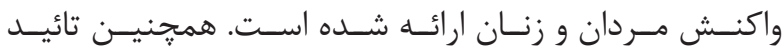

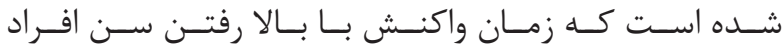

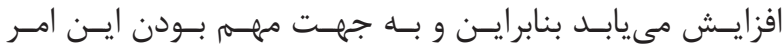

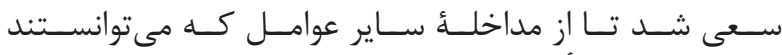

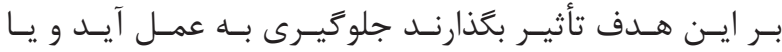

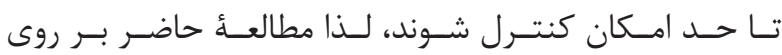

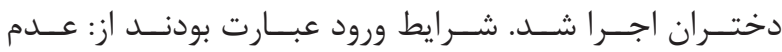

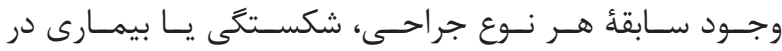

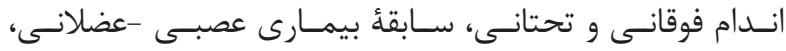

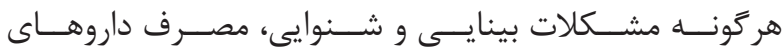

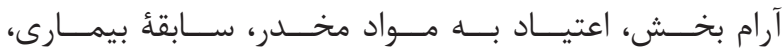

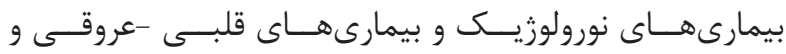

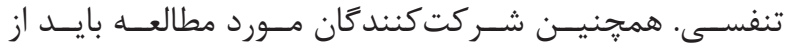
خ

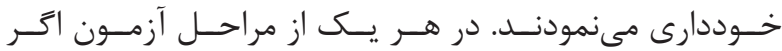

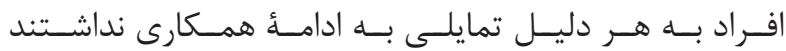

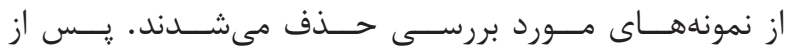

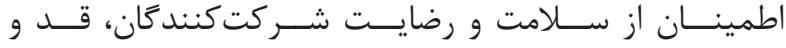

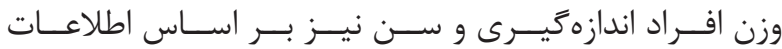

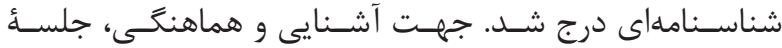

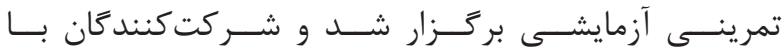

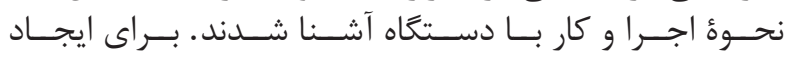

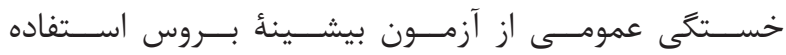

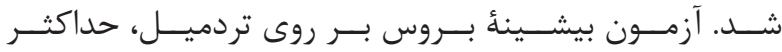

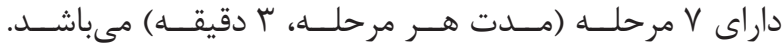

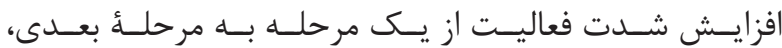

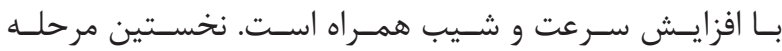

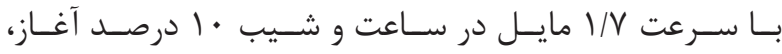

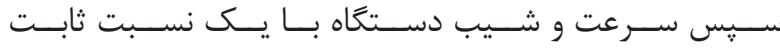

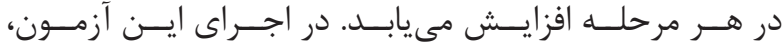

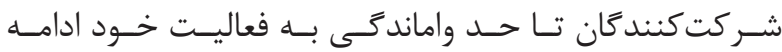

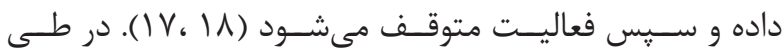




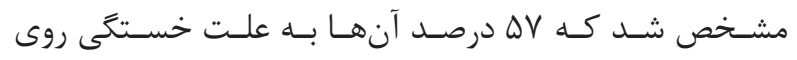

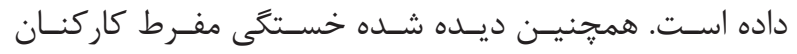

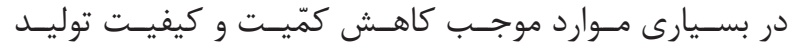

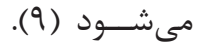

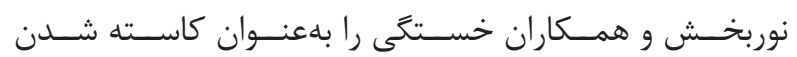

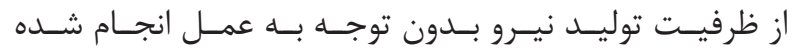

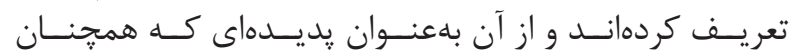

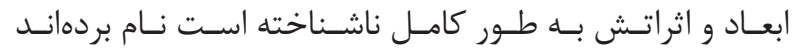

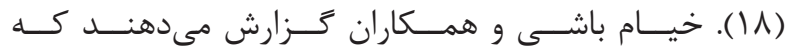

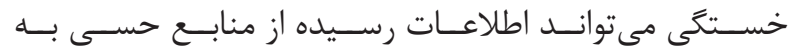

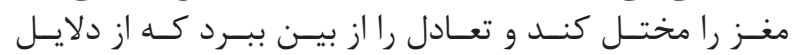

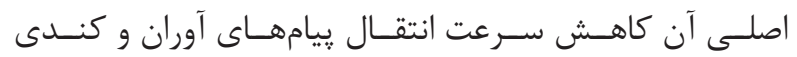

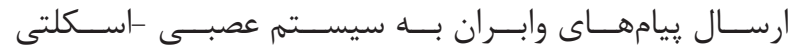

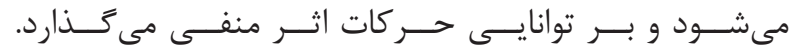

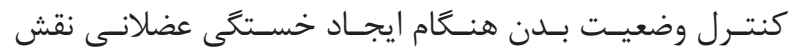

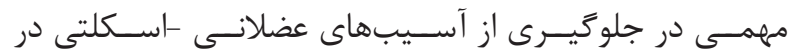

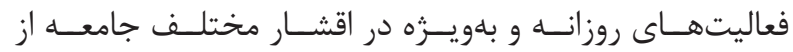

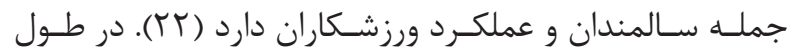

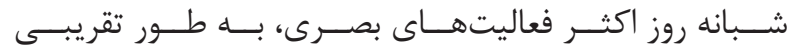

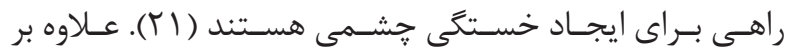

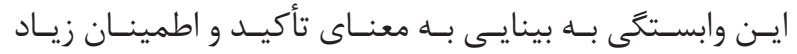

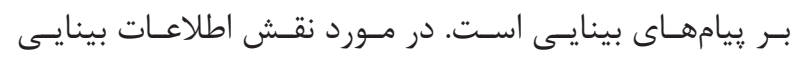

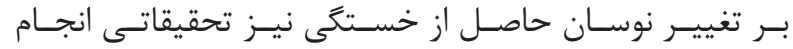

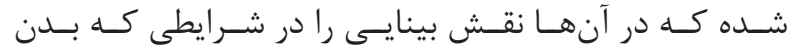

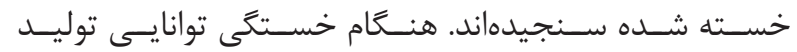

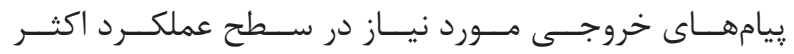

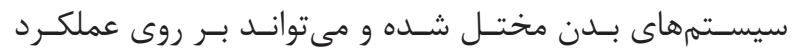

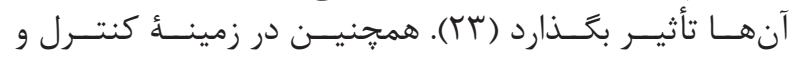

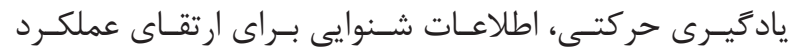

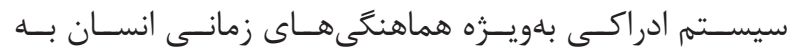

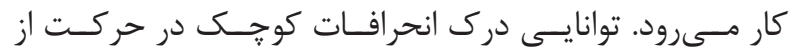

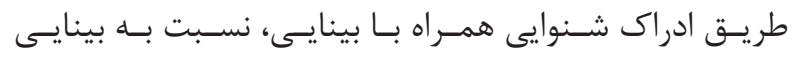

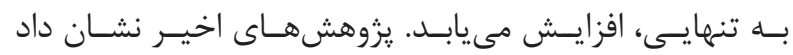

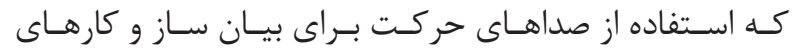

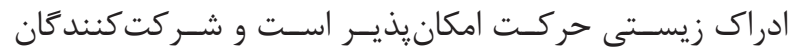

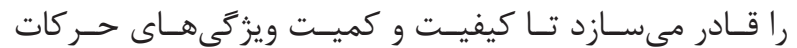

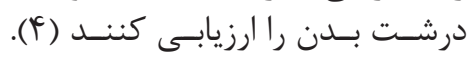

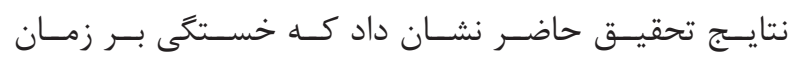

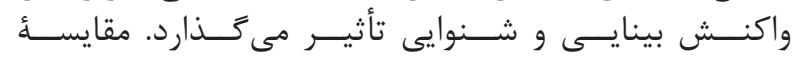

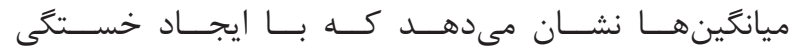

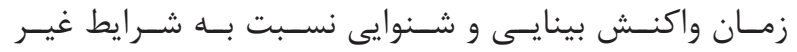

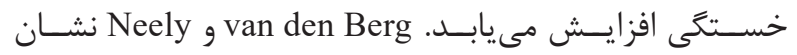

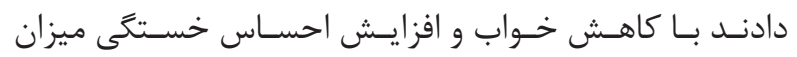

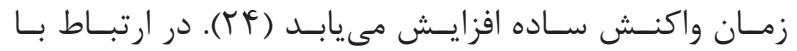

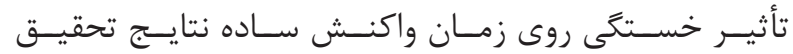
Ozdemir

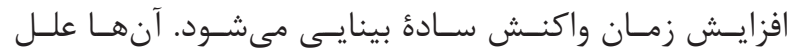

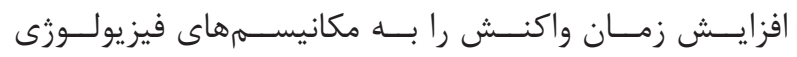

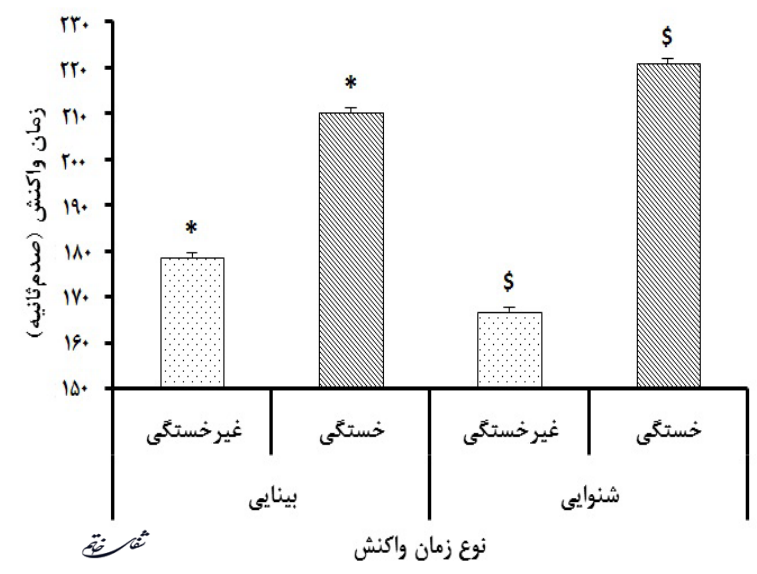

نمودار ا- ميانكين و انحراف استاندارد زمان واكنش بينايى و شنوايى در شرايط غير خستخى و خستى نى.

زمـان واكنـش بينايسى بـا شـــوايى آزمــون تـى مسـتقل بيـن

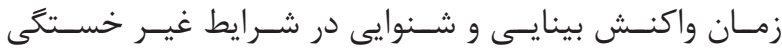

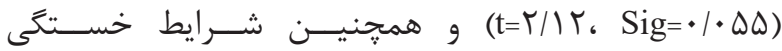

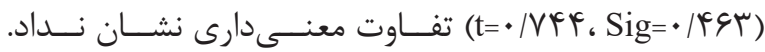

بحث و نتيجه كيرى

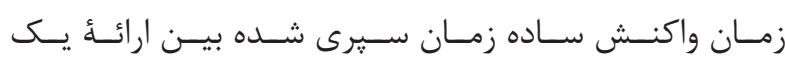

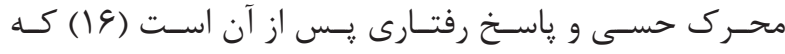

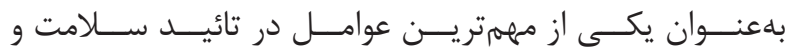

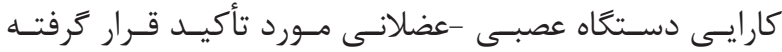

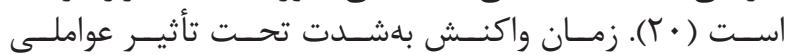

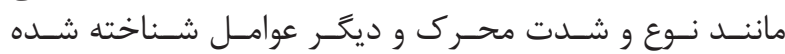

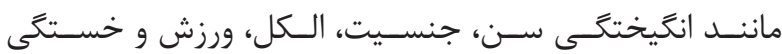

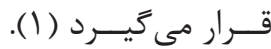

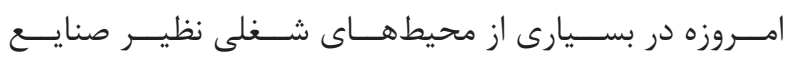

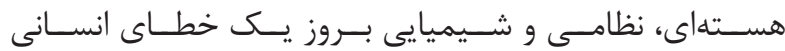

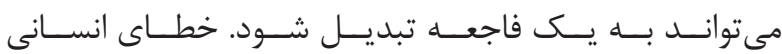

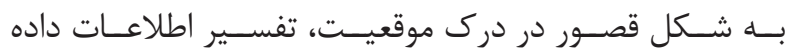

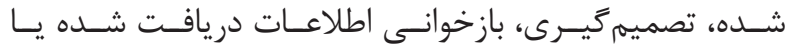

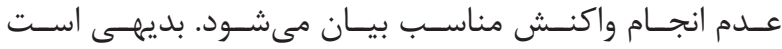

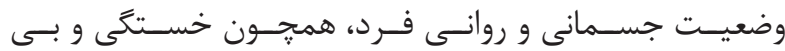

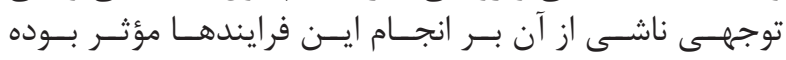

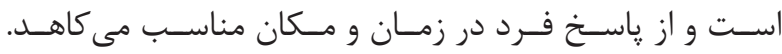

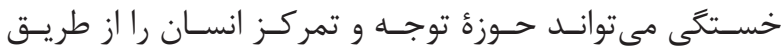

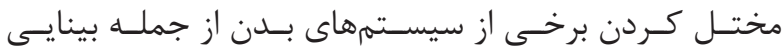

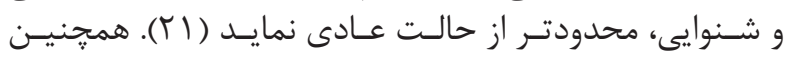

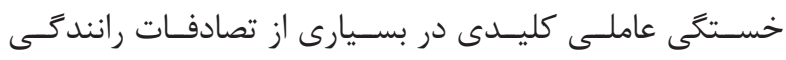

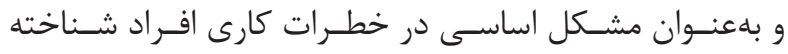

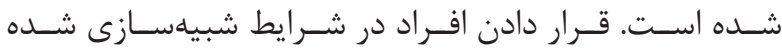

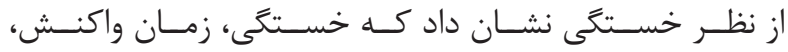

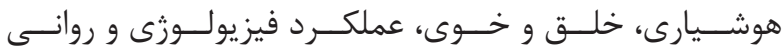

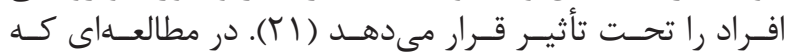

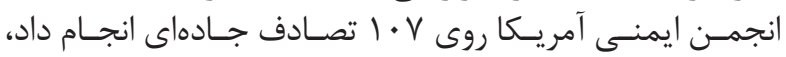




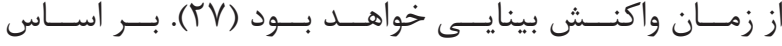

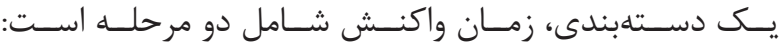

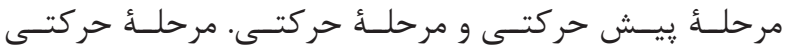

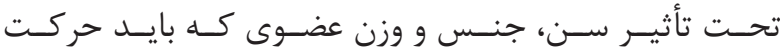

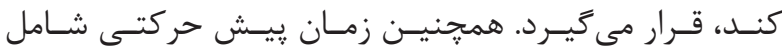

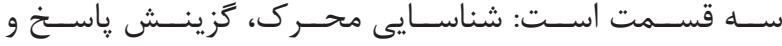

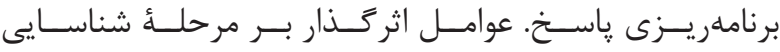

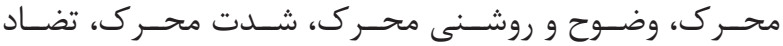

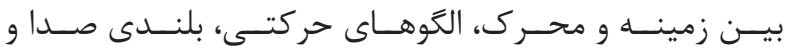

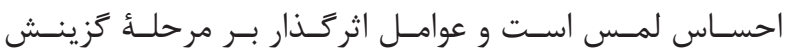

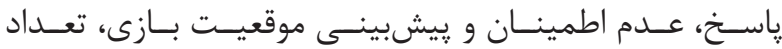

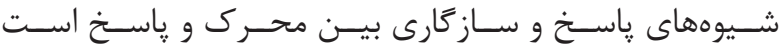
و يِيجيدگ

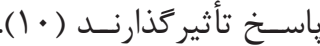

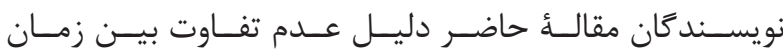

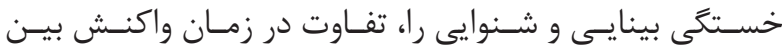

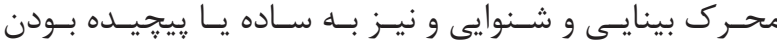

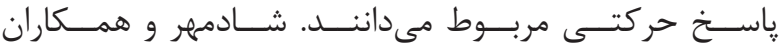

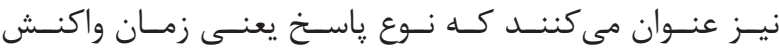

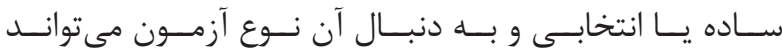

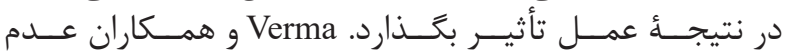

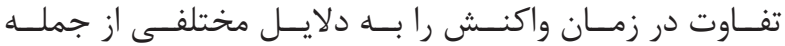

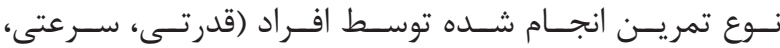

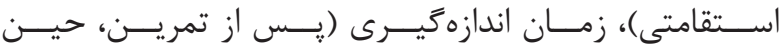

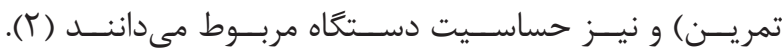

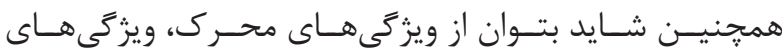

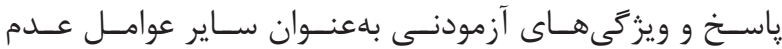

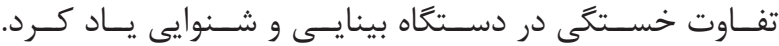

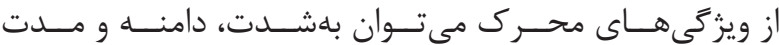

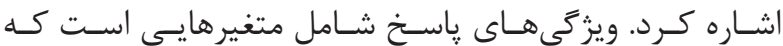

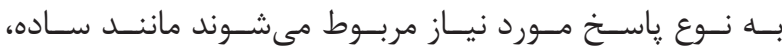

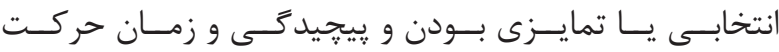

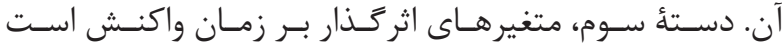

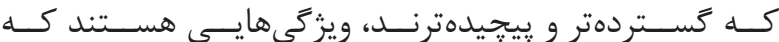

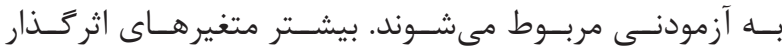

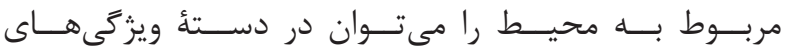

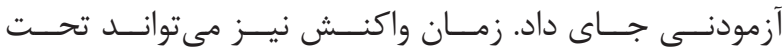

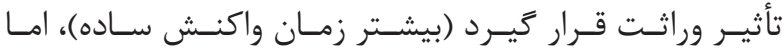

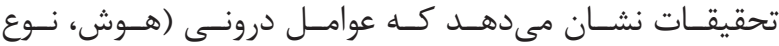

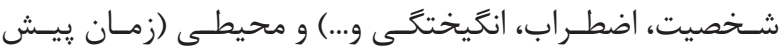

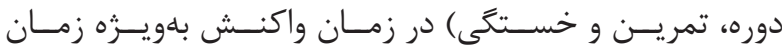

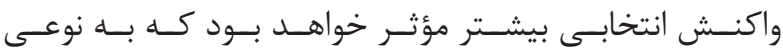

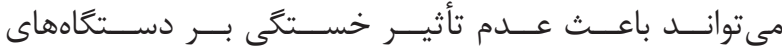

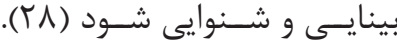

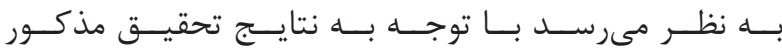

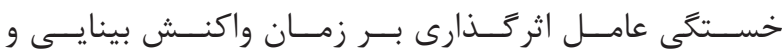

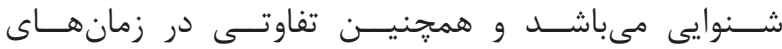

مختلــف نســبت مىدهنـــد (Tr).

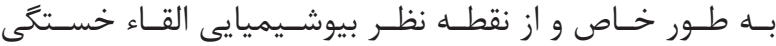

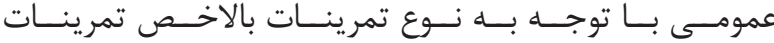

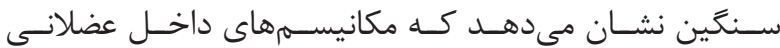

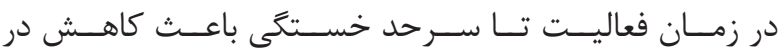

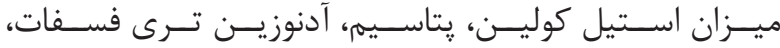

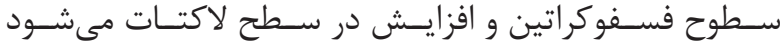

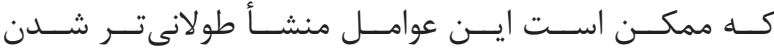

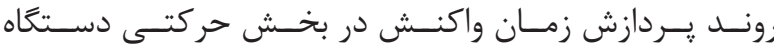

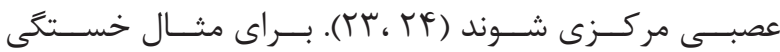

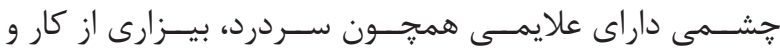

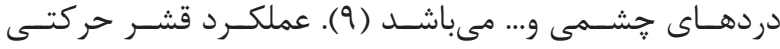

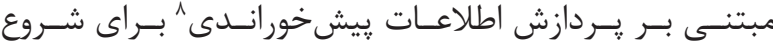

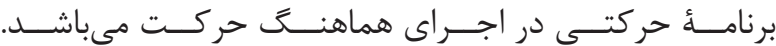

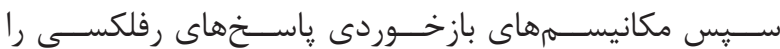

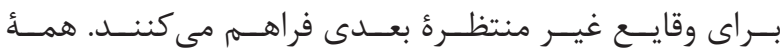

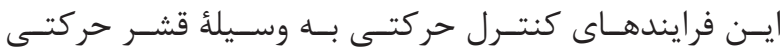

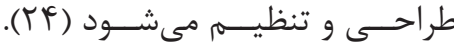

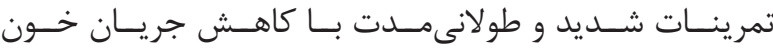

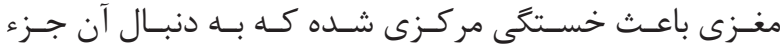

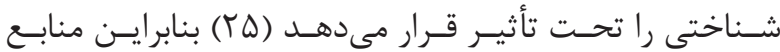

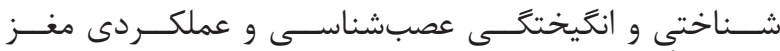

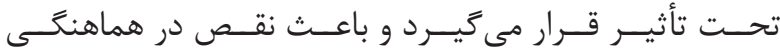

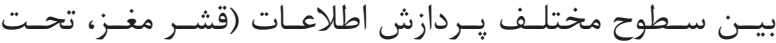

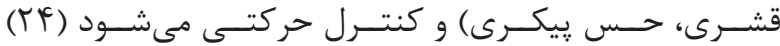

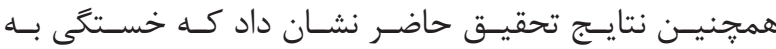

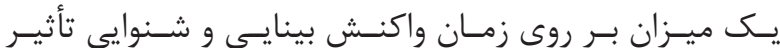

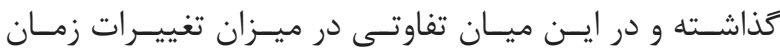

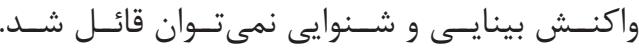

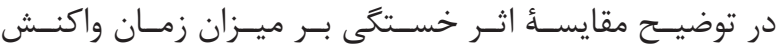

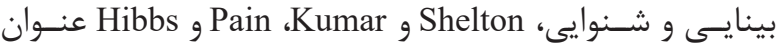

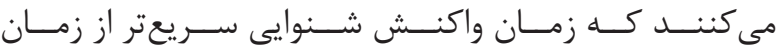

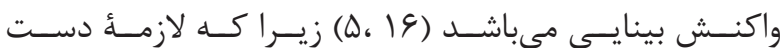

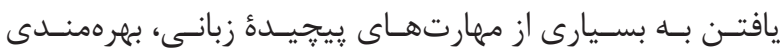

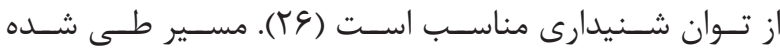

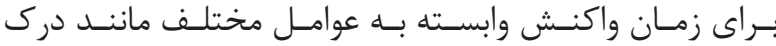

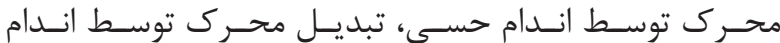

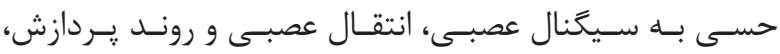

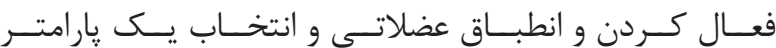

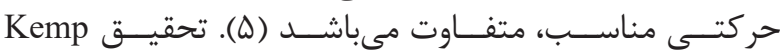

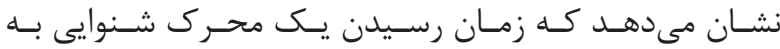

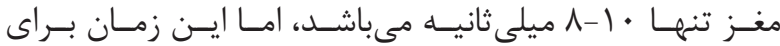

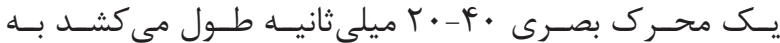

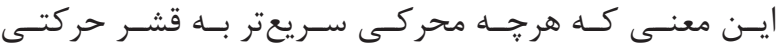

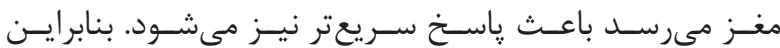

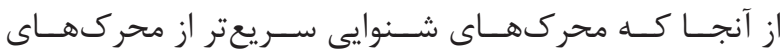

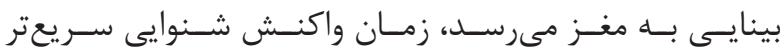

${ }^{8}$ Feed forward 


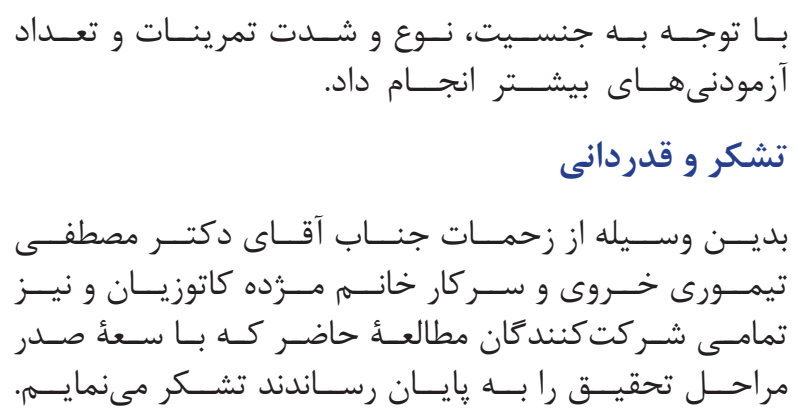

1. Garg M, Lata H, Walia L, Goyal O. Effect of aerobic exercise on auditory and visual reaction times: a prospective study. Indian J Physiol Pharmacol. 2013; 57(2): 138-45.

2. Verma SK, Mishra A, Singh A. Effect of long term physical exercise training on auditory and visual reaction time. Indian J Physiother Occup Ther. 2011; 5(3): 126-9.

3. Gavkare AM, Nanaware NL, Surdi AD. Auditory reaction time, visual reaction time and whole body reaction time in athletes. Ind Med Gaz. 2013; 147(6): 214-9.

4. Ramezanzade H, Abdoli B, Farsi A, Sanjari MA. The effect of audiovisual integration on performance accuracy and learning in motor task. J Res Rehab Sci. 2015; 11(1): 1-16.

5. Pain MT, Hibbs A. Sprint starts and the minimum auditory reaction time. J Sport Sci. 2007; 25(1): 79-86.

6. Thompson P, Colebatch J, Brown P, Rothwell J, Day $\mathrm{B}$, Obeso J, et al. Voluntary stimulus-sensitive jerks and jumps mimicking myoclonus or pathological startle syndromes. Movement Disorders. 1992; 7(3): 257-62.

7. Yagi Y, Coburn KL, Estes KM, Arruda JE. Effects of aerobic exercise and gender on visual and auditory P300, reaction time, and accuracy. Eur J Appl Physiol Occup Phys. 1999; 80(5): 402-8.

8. Verleger R. On the utility of P3 latency as an index of mental chronometry. Psychophysiology. 1997; 34(2): 131-56.

9. Arghami S, Ghoreishi A, Kamali K, Farhadi M. Investigating the consistency of mental fatigue measurements by visual analog scale (vas) and flicker fusion apparatus. Journal of Ergonomics. 2013; 1(1): 66-72.

10. Khajoei Ravari E, Farokhi A, Abas Gholi Pour A, Karshenas Najaf Abadi N, Soheilipour S. The effect of environmental color on simple reaction time to auditory stimulus. JMLD. 2013; 5(3): 27-40.

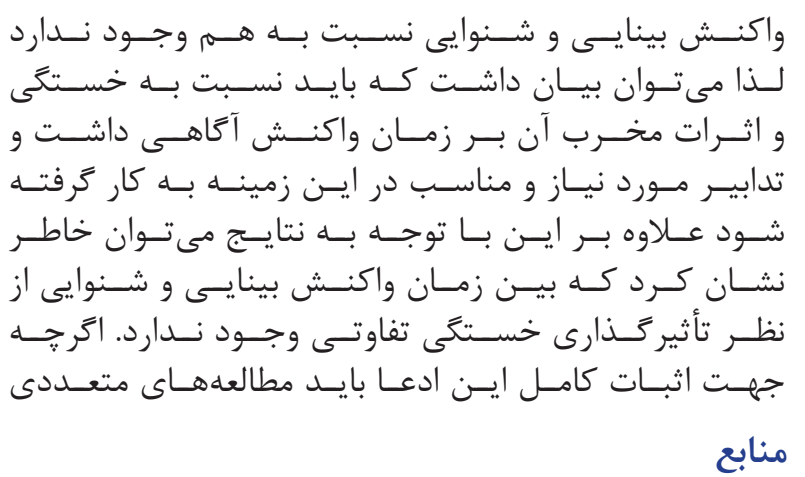

11. Fox EL, Bowers RW, Foss ML. The physiological basis of physical education and athletics. $4^{\text {th }}$. William C Brown Pub. 1989. p. 752.

12. Abdoli B, Farsi A, Tymuri M. The effect of mental pratice and physical pratice in fatigue and non- fatigue condition on the generalized motor program and parameter learning. J Sport Manage. 2014; 10(19): 25-38.

13. Ahmadi M, Akbari M, Dadgoo M, Talebian S, Pahnabi GR. The effect of lumbar muscle fatigue on postural control in Athlete and non-Athlete subjects. Modern Rehabilitation. 2015; 9(2): 8-15.

14. Latash ML. Neurophysiological basis of movement. $2^{\text {nd. }}$. Human Kinetics. 2008. p. 427.

15. Jason LA, Corradi K, Torres-Harding S, Taylor RR, King C. Chronic fatigue syndrome: the need for subtypes. Neuropsychol Rev. 2005; 15(1): 29-58.

16. Shelton J, Kumar GP. Comparison between auditory and visual simple reaction times. Neuroscience and Medicine. 2010; 1(1): 30-2.

17. Saghebjoo M, Dadi Khaliran Z, Afzalpour ME, Hedayati M, Yaghoubi A. Comparison of some prognostic markers of cardiovascular diseases to morning and evening Bruce treadmill test in women. J Birjand Univ Med Sci. 2013; 20(3): 252-61.

18. Nourbakhsh P, Sepasi H, Rezaee S. The effect of three different types of fatigue protocols on dynamic balance in female athlete students. Sport Sciences Quarterly. 2011; 2(6): 133-48.

19. Tofighi A, Saedmocheshi S. C-reactive protein and lactate response to consumption of sodium bicarbonate supplementation and exhaustive in young active. Journal of Kermanshah University of Medical Sciences. 2013; 17(2): 97-102.

20. Magill RA. Motor learning and control. Concepts and applications. 2004. p. 7.

21. Habibi E, Pourabdian S, Rajabi H, Dehghan H, 


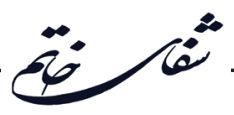

Maracy MR. Development and validation of a visual fatigue questionnaire for video display terminal users. HSR. 2011; 7(4): 492-503.

22. Khayambashi KH, Raziqi M, Abolqasm nzhad A, Mojtahedi $\mathrm{H}$. The effect of quadriceps muscle fatigue on dynamic balance while walking. Sports Medicine. 2010; 5: 35-49.

23. Ozdemir RA, Kirazci S, Uğraş A. Simple reaction time and decision making performance after different physical workloads: an examination with elite athletes. Journal of Human Sciences. 2010; 7(2): 655-70.

24. Van Den Berg J, Neely G. Performance on a simple reaction time task while sleep deprived 1,2. Perceptual and Motor Skills. 2006; 102(2): 589-99.

25. Ozyemisci-Taskiran O, Gunendi Z, Bolukbasi N,
Beyazova M. The effect of a single session submaximal aerobic exercise on premotor fraction of reaction time: an electromyographic study. Clinical Biomechanics. 2008; 23(2): 231-5.

26. Riyasi M, Sadat Dastgheib S. Utilization of basic concepts to accelerate language acquisition in children after cochlear lmplantation. Shefaye Khatam. 2013; 3: 49-53.

27. Kemp BJ. Reaction time of young and elderly subjects in relation to perceptual deprivation and signal-on versus signal-off conditions. Developmental Psychology. 1973; 8(2): 268-72.

28. Shahbazi M, Pashabad A, Parizi HA. Relationship between state and trait anxiety, reaction time and IQ in elite, sub-elite athletes and non-athletes. Journal of Development and Motor Learning (Harakat). 2012; 3(2): 65-80. 